\title{
Unsupervised gene network inference with decision trees and Random forests
}

\author{
Vân Anh Huynh-Thu and Pierre Geurts
}

\begin{abstract}
In this chapter, we introduce the reader to a popular family of machine learning algorithms, called decision trees. We then review several approaches based on decision trees that have been developed for the inference of gene regulatory networks (GRNs). Decision trees have indeed several nice properties that make them well-suited for tackling this problem: they are able to detect multivariate interacting effects between variables, are non-parametric, have good scalability, and have very few parameters. In particular, we describe in detail the GENIE3 algorithm, a state-of-the-art method for GRN inference.
\end{abstract}

Key words: Machine learning, decision trees, regression trees, tree ensembles, Random forest

\section{Introduction}

This chapter focuses on a popular family of machine learning algorithms, called decision trees. The goal of tree-based algorithms is to learn a model, in the form of a decision tree or an ensemble of decision trees, that is able to predict the value of an output variable given the values of some input variables. Tree-based methods have been widely used to solve diverse problems in computational biology, such as DNA sequence annotation or biomarker discovery (see [1-3] for reviews). In particular, several approaches based on decision trees have been developed for the inference of gene regulatory networks (GRNs) from expression data. Decision trees have indeed several advantages that make them attractive for tackling this problem. First, they are potentially able to detect multivariate interacting effects between variables, which make them well suited for modelling gene regulation, as the regulation of the expres-

Vân Anh Huynh-Thu · Pierre Geurts

Department of Electrical Engineering and Computer Science, University of Liège, Liège, Belgium e-mail: vahuynh@uliege.be, p.geurts@uliege.be 
sion of one gene is expected to be combinatorial, i.e. to involve several regulators. Tree-based methods have also the advantage to be non-parametric. They thus do not make any assumption about the nature of the interactions between the variables (such as linearity or Gaussianity). As their computational complexity is typically at most linear in the number of features, they can deal with high-dimensionality, a characteristic usually encountered in gene expression datasets. They are also flexible as they can handle both continuous and discrete variables. With respect to other supervised learning methods such as support vector machines or artificial neural networks, tree-based methods have very few parameters, which make them easy to use, even for non-specialists. See also Chapter 9 for another usage of tree-based methods in GRN inference.

One of the most widely used tree-based methods for GRN inference is GENIE3 [4]. This method exploits variable importance scores derived from ensembles of regression trees to identify the regulators of each target gene. GENIE3 was the best performer of the DREAM4 Multifactorial Network challenge and the DREAM5 Network Inference challenge [5], and is currently one of the state-of-the-art approaches for GRN inference. This method has also been evaluated and compared to other methods in numerous independent studies (e.g. [6-13]), usually achieving competitive results, and has often been used (either alone or in combination with other inference methods) to reconstruct real networks in various organisms such as bacteria [14-16], plants [17-19], drosophila [20], mouse [21, 22], and human $[23,24]$.

The chapter is structured as follows. Section 2 introduces general notions of supervised learning, while Section 3 specifically focuses on regression tree-based approaches. Section 4 presents several tree-based methods for GRN inference. In particular, the GENIE3 algorithm is described in detail. Finally, Section 5 discusses potential improvements of GENIE3.

\section{Supervised learning}

Machine learning is a branch of artificial intelligence whose goal is to extract knowledge from observed data. In particular, supervised learning is the machine learning task of inferring a model $f$ that predicts the value of an output variable $Y$, given the values of $m$ inputs $X_{1}, X_{2}, \ldots, X_{m}$. The model $f$ is learned from $N$ instances (also called samples or observations) of input-output pairs, drawn from the (usually unknown) joint distribution $p\left(X_{1}, X_{2}, \ldots, X_{m}, Y\right)$ of the variables:

$$
L S=\left\{\left(\mathbf{x}_{k}, y_{k}\right)\right\}_{k=1}^{N} .
$$

The set of instances is called learning sample. Depending on whether the output is discrete or continuous, the learning problem is a classification or a regression problem respectively. In this chapter, we will focus on regression problems. 
Let $L$ be a loss function that, given an instance $(\mathbf{x}, y)$, measures the difference between the value $f(\mathbf{x})$ predicted by the model $f$ from the input $\mathbf{x}$, and the observed value $y$ of the target variable. For a regression problem, a widely used loss function is the squared error:

$$
L(y, f(\mathbf{x}))=(y-f(\mathbf{x}))^{2} .
$$

The goal of supervised learning is to find, from a learning sample $L S$, a model $f$ that minimises the generalisation error, i.e. the expected value of the loss function, taken over different instances randomly drawn from the joint distribution $p\left(X_{1}, X_{2}, \ldots, X_{m}, Y\right)$ :

$$
E_{\mathbf{x}, y}[L(y, f(\mathbf{x}))] .
$$

Since the joint distribution $p\left(X_{1}, X_{2}, \ldots, X_{m}, Y\right)$ is usually unknown, supervised learning algorithms typically work by minimising the training error, which is the average prediction error of the model over the instances of the learning sample:

$$
\frac{1}{N} \sum_{k=1}^{N} L\left(y_{k}, f\left(\mathbf{x}_{k}\right)\right)
$$

As the training error is calculated on the same samples that were used to learn the predictive model, it typically underestimates the generalisation error, as shown in Figure 1. The training error typically decreases when the complexity of the model is increased, i.e. when the model is allowed to fit more closely the training data. If the complexity is too high, the model may also fit the noise contained in the data and thus will have a poor generalisation performance. In this case, we say that the model overfits the training data. On the other hand, if the model has a too low complexity, it underfits the data and will also have a high generalisation error. Hence there is an optimal model complexity that leads to the minimal generalisation error.

For more details, the reader is invited to refer to general books about machine learning $[25,26]$.

\section{Regression trees}

A popular approach to the regression problem is the regression tree [27]. Figure 2 shows the structure of a tree. In this example, there are two input variables $X_{1}$ and $X_{2}$, which are both continuous. Each interior node of the tree contains a test of the type " $X_{i}<c$ ", where $X_{i}$ is one of the input variables and $c$ a threshold value, and each terminal node (or leaf) contains a predicted value for the output variable. Given a new sample, for which we have observed values of the input variables, a prediction for the output is obtained by propagating the sample down the tree, until it reaches a leaf. The predicted output value is then the value at that leaf. 


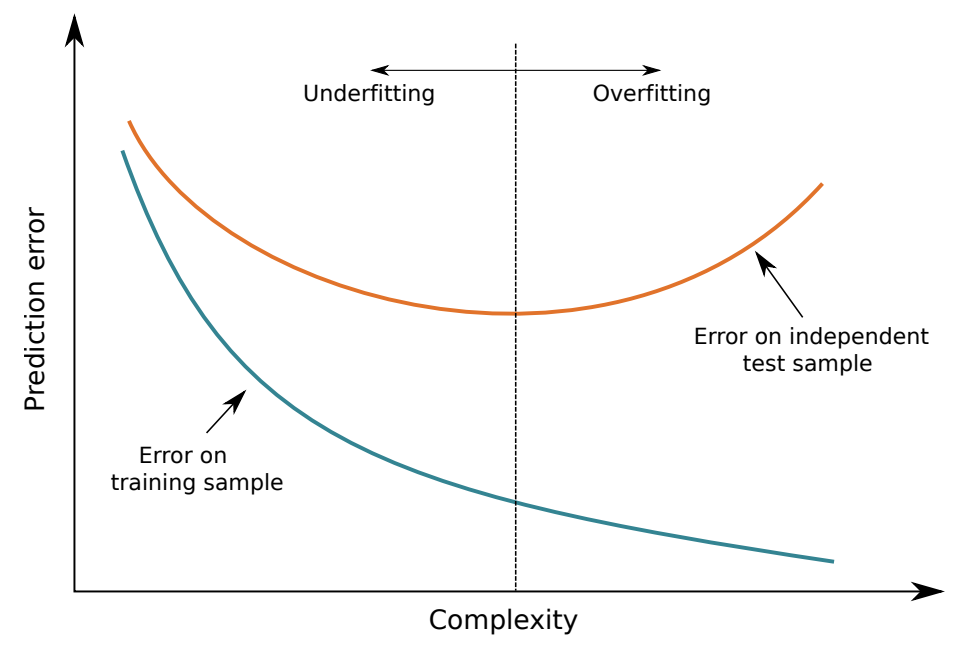

Fig. 1 Overfitting and underfitting. The blue (resp. orange) curve plots, for varying levels of complexity of the predictive model, the average value of the loss function over the instances of the learning sample (resp. of an independent test sample). Overfitting occurs when the model is too complex and underfitting occurs when the model is not complex enough.

Fig. 2 Example of a regression tree. Each interior node of a tree is a test on one input variable and each terminal node contains a predicted value for the output variable.

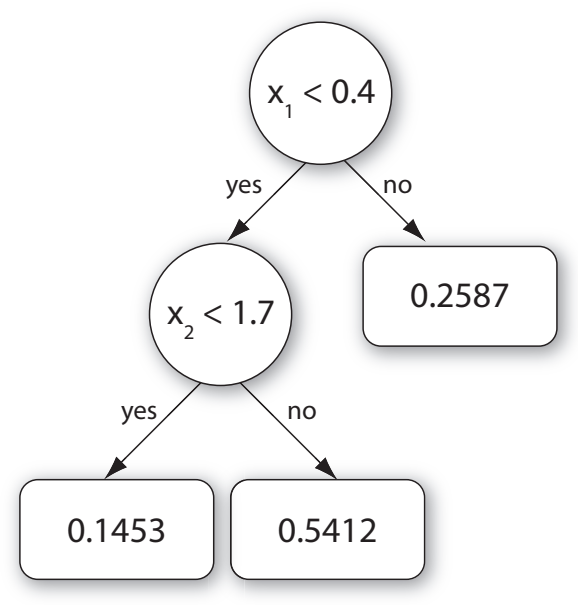

\subsection{Learning a regression tree}

Using a learning sample $L S$, the goal of a tree-based method is to identify the tree that minimises the training error in Equation (4). A brute-force approach would consist in enumerating all the possible trees. This approach is however intractable and for this reason tree-based methods are rather based on greedy algorithms. A regression tree is typically constructed top-down, starting from a root node corresponding to the whole learning sample. The idea is then to recursively split the learning sam- 
ple with binary tests on the values of the input variables, trying to reduce as much as possible the variance of the output variable in the resulting subsets of samples. At each interior node $\mathscr{N}$, the best test " $X_{i}<c$ " is chosen, i.e. the variable $X_{i}$ and the threshold value $c$ that maximise:

$$
I(\mathscr{N})=\# S \cdot \operatorname{Var}_{Y}(S)-\# S_{t} \cdot \operatorname{Var}_{Y}\left(S_{t}\right)-\# S_{f} \cdot \operatorname{Var}_{Y}\left(S_{f}\right),
$$

where $S$ denotes the set of samples of $L S$ that reach node $\mathscr{N}, S_{t}$ (resp. $S_{f}$ ) denotes its subset for which the test is true (resp. false), \# denotes the cardinality of a set of samples, and $\operatorname{Var}_{Y}(\cdot)$ is the variance of the output in a subsample. The samples of $S$ are then split into two subsamples following the optimal test and the same procedure is applied on each of these subsamples. A node becomes a terminal node if the variance of the output variable, computed over the samples reaching that node, is equal to zero. Each terminal node contains a predicted value for the output, corresponding to the mean value of the output taken over the samples that reach that node.

However, a fully grown tree typically overfits the training data. Overfitting can be avoided by pruning the tree, i.e. by removing some of its subtrees. Two types of pruning exist: pre-pruning and post-pruning. In a pre-pruning procedure, a node becomes a terminal node instead of a test node if it meets a given criterion, such as:

- The number of samples reaching the node is below a threshold $N_{\min }$;

- The variance of the output variable, over the samples reaching the node, is below a threshold Var $_{\text {min }}$;

- The optimal test is not statistically significant, according to some statistical test.

On the other side, the post-pruning procedure consists in fully developing a first tree $\mathscr{T}_{1}$ from the learning sample and then computing a sequence of trees $\left\{\mathscr{T}_{2}, \mathscr{T}_{3}, \ldots\right\}$ such that $\mathscr{T}_{i}$ is a pruned version of $\mathscr{T}_{i-1}$. The prediction error of each tree is then calculated on an independent set of samples and the tree that leads to the lowest prediction error is selected. The main drawback of the post-pruning procedure is that an independent set of samples is needed, while the main drawback of pre-pruning is that the optimal value of the parameter related to the chosen stop-splitting criterion ( $N_{\min }, \operatorname{Var}_{\min }$, the significance level) is dependent on the considered problem.

Besides pruning, ensemble methods constitute another way of avoiding overfitting. These methods are described in the following section.

\subsection{Ensemble methods}

Single regression trees are usually very much improved by ensemble methods, which average the predictions of several trees. The goal of ensemble methods is to use diversified models to reduce the overfitting of a learning algorithm. In the case of a tree, the overfitting comes mostly from the choices, made at each split node, of the input variable and the threshold value used for the test. Amongst the most widely used tree-based ensemble methods are methods that rely on randomi- 
sation to generate diversity among the different models. These methods are Bagging [28], Random forest [29], and Extra-Trees [30].

Bagging

In the Bagging (for "Bootstrap AGGregatING") algorithm, each tree of the ensemble is built from a bootstrap replica, i.e. a set of samples obtained by $N$ random samplings with replacement in the original learning sample. The choices of the variable and of the threshold at each test node are thus implicitly randomised via the bootstrap sampling.

Random forest

This method adds an extra level of randomisation compared to the Bagging. In a Random forest ensemble, each tree is built from a bootstrap sample of the original learning sample and at each test node, $K$ variables are selected at random (without replacement) among all the input variables before determining the best split. When $K$ is set to the total number of input variables, the Random forest algorithm is equivalent to Bagging.

\section{Extra-Trees}

In the Extra-Trees (for "EXTremely RAndomised Trees") method, each tree is built from the original learning sample but at each test node, the best split is determined among $K$ random splits, each determined by randomly selecting one input variable (without replacement) and a threshold value (chosen uniformly between the minimum and maximum values of the input variable in the local subset of samples).

\subsection{Parameters}

Tree-based (ensemble) methods have several parameters whose values must be set by the user:

- The parameters related to the chosen stop-splitting criterion, such as $N_{\min }$, the minimal number of samples that a leaf node must contain. Increasing the value of $N_{\min }$ results in smaller trees and hence models with a higher bias (i.e. more prone to underfitting) and a lower variance (i.e. less prone to overfitting). Its optimal value depends on the level of noise contained in the learning sample. The noisier the data, the higher the optimal value of $N_{\min }$. Usually, $N_{\min }$ is fixed to 1 for ensemble methods, so that each tree of the ensemble is fully developed. 
- $K$, the number of input variables that are randomly chosen at each node of a tree. This parameter thus determines the level of randomisation of the trees. A smaller value of $K$ results in more randomised trees. The optimal value of $K$ is problemdependent, but $K=\sqrt{m}$ and $K=m$, where $m$ is the number of input variables, are usually good default values [30].

- $T$, the number of trees in an ensemble. It can be shown that the higher the number of trees, the lower the generalisation error [29,3]. Therefore, the chosen value of $T$ is a compromise between model accuracy and computing times.

\subsection{Variable importance measures}

One interesting characteristic of tree-based methods is the possibility to compute from a tree an importance score for each input variable. This score measures the relevance of a variable for the prediction of the output. In the case of regression, an importance measure that can be used is based on the reduction of the variance of the output at each test node $\mathscr{N}$, i.e. $I(\mathscr{N})$ as defined in Equation (5). For a single tree, the overall importance $w_{i}$ of one variable $X_{i}$ is then computed by summing the $I(\mathscr{N})$ values of all the tree nodes where $X_{i}$ is used to split:

$$
w_{i}=\sum_{k=1}^{p} I\left(\mathscr{N}_{k}\right) \mathbb{1}_{\mathscr{N}_{k}}\left(X_{i}\right),
$$

where $p$ is the number of test nodes in the tree and $\mathscr{N}_{k}$ denotes the $k$-th test node. $\mathbb{1}_{\mathscr{N}_{k}}\left(X_{i}\right)$ is a function that is equal to one if $X_{i}$ is the variable selected at node $\mathscr{N}_{k}$ and zero otherwise. The features that are not selected at all thus obtain an importance value of zero and those that are selected close to the root node of the tree typically obtain high scores. Variable importance measures can be easily extended to ensembles, simply by averaging importance scores over all the trees of the ensemble. The resulting importance measure is then even more reliable because of the variance reduction effect resulting from this averaging.

In the context of the Random forest method, an alternative procedure was proposed to compute the importance of a variable [29]. For each tree that was learned, this procedure consists in computing the prediction accuracy of the tree on the outof-bag samples (i.e. the training instances that were not present in the bootstrap sample used to build the tree), before and after randomly permuting the values of the corresponding variable in these samples. The reduction of the tree accuracy that is obtained after the permutation is then computed, and the importance of the variable is given by the average accuracy reduction over all the trees of the ensemble. While this procedure has some advantages with respect to the variance reductionbased measure [31], it gives in most practical applications very similar results while being much more computationally demanding. Furthermore, it does not extend to methods that do not consider bootstrap sampling, like the Extra-Trees. 


\section{Tree-based approaches for gene network inference}

This section presents several approaches based on decision tree algorithms, that were developed for the unsupervised inference of GRNs. In particular, we start by describing in detail the state-of-the-art GENIE3 approach.

\subsection{GENIE3}

GENIE3, for "GEne Network Inference with Ensemble of trees", uses ensembles of regression trees to infer GRNs from steady-state expression data. (Many people think that the digit 3 in the acronym GENIE3 indicates a third version of the algorithm. This is however not the case. The presence of the digit 3 is actually due to the fact that the word "three" sounds exactly like the word "tree", when pronounced with a (strong) French accent.)

In what follows, we define an expression dataset from which to infer the network as a collection of $N$ measurements:

$$
D=\left\{\mathbf{x}_{1}, \mathbf{x}_{2}, \ldots, \mathbf{x}_{N}\right\}
$$

where $\mathbf{x}_{k} \in \mathbb{R}^{G}, k=1, \ldots, N$ is the vector of expression values of $G$ genes in the $k$-th experiment:

$$
\mathbf{x}_{k}=\left(x_{k}^{1}, x_{k}^{2}, \ldots, x_{k}^{G}\right)^{\top} .
$$

The goal of GENIE3 is to exploit the expression dataset $D$ to assign weights $w_{i, j}>0,(i, j=1, \ldots, G)$ to putative regulatory links from any gene $g_{i}$ to any gene $g_{j}$, with the aim of yielding larger values for weights that correspond to actual regulatory interactions. GENIE3 returns directed and unsigned edges, which means that $w_{i, j}$ can take a different value than $w_{j, i}$, and when $g_{i}$ is connected to $g_{j}$, the former can be either an activator or a repressor of the latter.

To solve the network inference problem, GENIE3 decomposes the problem of recovering a network of $G$ genes into $G$ different subproblems, where each of these subproblems consists in identifying the regulators of one of the genes of the network. The method makes the assumption that the expression of each gene in a given condition is a function of the expression of the other genes in the same condition (plus some random noise). Denoting by $\mathbf{x}_{k}^{-j}$ the vector containing the expression values in the $k$-th experiment of all the genes except gene $g_{j}$ :

$$
\mathbf{x}_{k}^{-j}=\left(x_{k}^{1}, \ldots, x_{k}^{j-1}, x_{k}^{j+1}, \ldots, x_{k}^{G}\right)^{\top},
$$

we can write:

$$
x_{k}^{j}=f_{j}\left(\mathbf{x}_{k}^{-j}\right)+\varepsilon_{k}, \forall k,
$$

where $\varepsilon_{k}$ is a random noise with zero mean (conditionally to $\mathbf{x}_{k}^{-j}$ ). GENIE3 further makes the assumption that the function $f_{j}$ only exploits the expression in $\mathbf{x}^{-j}$ of the 
genes that are direct regulators of $g_{j}$, i.e. genes that are directly connected to $g_{j}$ in the targeted network. Recovering the regulatory links pointing to the target gene $g_{j}$ thus amounts to finding those genes whose expression is predictive of the expression of $g_{j}$. In machine learning terminology, this can be considered a feature selection problem (in regression) for which many solutions can be found in the literature. The solution that is used by GENIE3 exploits the variable importance scores derived from tree ensemble models.

The GENIE3 procedure is illustrated in Figure 3 and works as follows:

- For $j=1$ to $G$ :

- Generate the learning sample of input-output pairs for gene $g_{j}$ :

$$
L S^{j}=\left\{\left(\mathbf{x}_{k}^{-j}, x_{k}^{j}\right), k=1, \ldots, N\right\} .
$$

- Learn an ensemble of trees from $L S^{j}$ using the Random forest or Extra-Trees algorithm.

- From the learned tree model, compute variable importance scores $w_{i, j}$ for all the genes $g_{i}$ (except $g_{j}$ itself). These importance scores are computed as sums of variance reductions (Equation (6)).

- Use $w_{i, j}$ as weight for the regulatory link directed from $g_{i}$ to $g_{j}$.

Note that in GENIE3, it is possible - and even advisable - to restrict the set of candidate regulators to a subset of the genes only (rather than using all the $G$ genes as candidate regulators). This can be useful when we know which genes are transcription factors for example. In that case, the learning sample $L S^{j}$ is constructed with only those transcription factors as input genes.

\subsubsection{Output normalisation}

In the GENIE3 procedure, each tree-based model yields a separate ranking of the genes as potential regulators of a target gene $g_{j}$, derived from importance scores $w_{i, j}$. It can be shown that the sum of the importance scores of all the input variables for a tree is equal to the total variance of the output variable explained by the tree, which in the case of unpruned trees (as they are in the case of tree ensembles) is usually very close to the initial total variance of the output:

$$
\sum_{i \neq j} w_{i, j} \approx N \operatorname{Var}_{j}\left(L S^{j}\right)
$$

where $L S^{j}$ is the learning sample from which the tree was built (i.e. $L S^{j}$ in the case of the Extra-Trees method and a bootstrap sample in the case of the Bagging and Random forest methods) and $\operatorname{Var}_{j}\left(L S^{j}\right)$ is the variance of the target gene $g_{j}$ estimated in the corresponding learning sample. As a consequence, if the regulatory links are simply ranked according to the weights $w_{i, j}$, this is likely to introduce a bias where some putative regulatory links will have a high weight simply because 


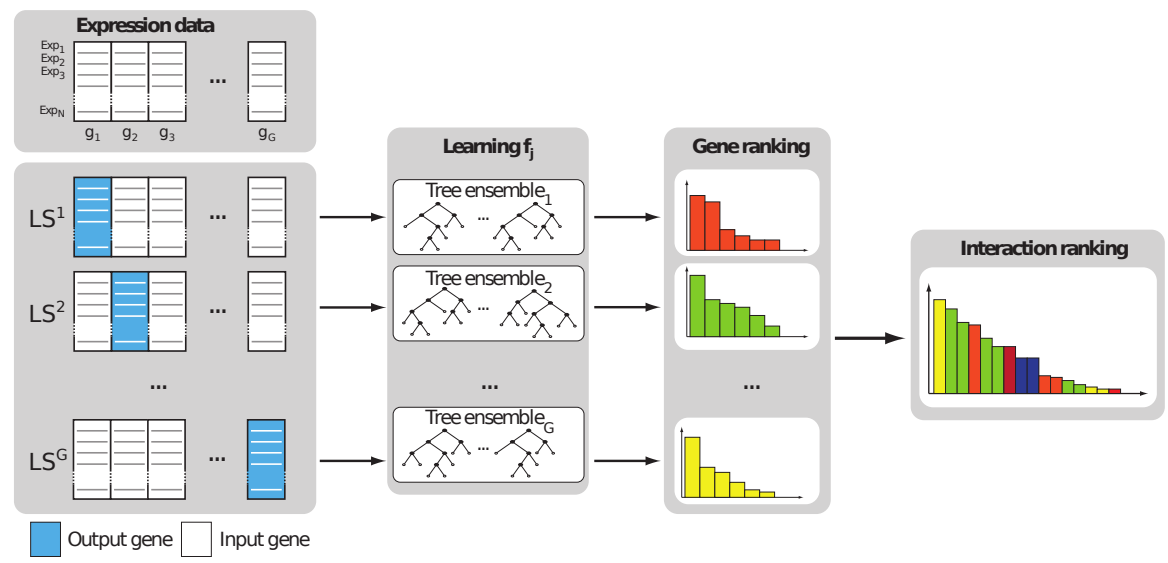

Fig. 3 GENIE3 procedure. For each gene $g_{j}, j=1, \ldots, G$, a learning sample $L S^{j}$ is generated with the expression levels of $g_{j}$ as output values and the expression levels of all the other genes as input values. An ensemble of trees is learned from $L S^{j}$ and a variable importance score $w_{i, j}$ is computed for each input gene $g_{i}$. The score $w_{i, j}$ is then used as weight for the regulatory link directed from $g_{i}$ to $g_{j}$. Figure reproduced from [4].

they are directed towards highly variable genes. To avoid this bias, the expression of the target gene $g_{j}$ is normalised to have a unit variance in the learning sample $L S^{j}$, before applying the tree-based ensemble method:

$$
\mathbf{x}^{j} \leftarrow \frac{\mathbf{x}^{j}}{\sigma^{j}}, \forall j
$$

where $\mathbf{x}^{j} \in \mathbb{R}^{N}$ is the vector of expression levels of $g_{j}$ in the $N$ experiments and $\sigma^{j}$ denotes its standard deviation. This normalisation indeed implies that the different weights inferred from different models predicting the different gene expressions are comparable.

\subsubsection{Software availability}

Python, MATLAB and R implementations of GENIE3, as well as tutorials explaining how to run them, are available from:

http://www.montefiore.ulg.ac.be/ huynh-thu/GENIE3.html

\subsubsection{Computational complexity}

The computational complexity of the Random forest and Extra-Trees algorithms is on the order of $O(T K N \log N)$, where $T$ is the number of trees, $N$ is the learning sample size, and $K$ is the number of randomly selected genes at each node of a tree. 
GENIE3's complexity is thus on the order of $O(G T K N \log N)$ since it requires to build an ensemble of trees for each of the $G$ genes. The complexity of the whole procedure is thus log linear with respect to the number of measurements and, at worst, quadratic with respect to the number of genes (when $K=G-1$ ).

To give an idea of computing times, Table 1 shows the times needed for GENIE3 to infer one network of the DREAM4 Multifactorial challenge (100 experiments and 100 genes) and the E. coli network of the DREAM5 challenge (805 experiments and 4511 genes, among which 334 known transcription factors). In each case, GENIE3 was run with Random forest, $T=1000$ trees per ensemble and $K=\sqrt{n_{T F}}$, where $n_{T F}$ is the number of candidate regulators (i.e. $n_{T F}=100$ for DREAM4 and $n_{T F}=$ 334 for E. coli). These computing times were measured on a 16GB RAM, Intel Xeon E5520 $2.27 \mathrm{GHz}$ computer.

Table 1 Running times of the different GENIE3 implementations

\begin{tabular}{llll}
\hline Network & Python & MATLAB & $\mathrm{R}$ \\
\hline DREAM4 $\left(N=100, G=100, n_{T F}=100\right)$ & $130 \mathrm{sec}$ & $65 \mathrm{sec}$ & $50 \mathrm{sec}$ \\
E. $\operatorname{coli}\left(N=805, G=4511, n_{T F}=334\right)$ & 20 hours & 20 hours & 14 hours \\
\hline
\end{tabular}

$N$ : number of samples, $G$ : number of genes, $n_{T F}$ : number of transcription factors.

Note that if needed, the GENIE3 algorithm can be easily parallelised as the $G$ feature selection problems, as well as the different trees in an ensemble, are independent of each other.

\subsubsection{Parameter analysis}

Figure 4 shows the performances and running times of GENIE3, for two networks of the DREAM5 challenge (an artificial In silico network and a real E. coli network), when varying the values of the different parameters of GENIE3. The performances were measured using the area under the precision-recall curve (AUPR) metric, which assesses the quality of the ranking of interactions returned by a method. A perfect ranking, where all the true interactions are ranked at the top, yields an AUPR equal to 1, while a random ranking returns an AUPR equal to the proportion of true interactions among all the possible interactions (which is typically very small, since regulatory networks are very sparse).

Clearly, the parameter with the highest impact is $K$, i.e. the number of randomly selected candidate regulators at each tree node. Its optimal value is very datasetdependent: increasing the value of $K$ improves the predictions (i.e. the AUPR is increased) for the In silico network, while the opposite is observed on the E. coli network. This difference between the two networks can probably be explained by the fact that the $E$. coli data contains more noise than the artificial data. We thus checked how the performance of GENIE3 varies when adding further noise to the artificial data, in the form of a Gaussian noise $\sim \mathscr{N}(0,0.25)$ or $\mathscr{N}(0,0.5)$ (Table 2). 

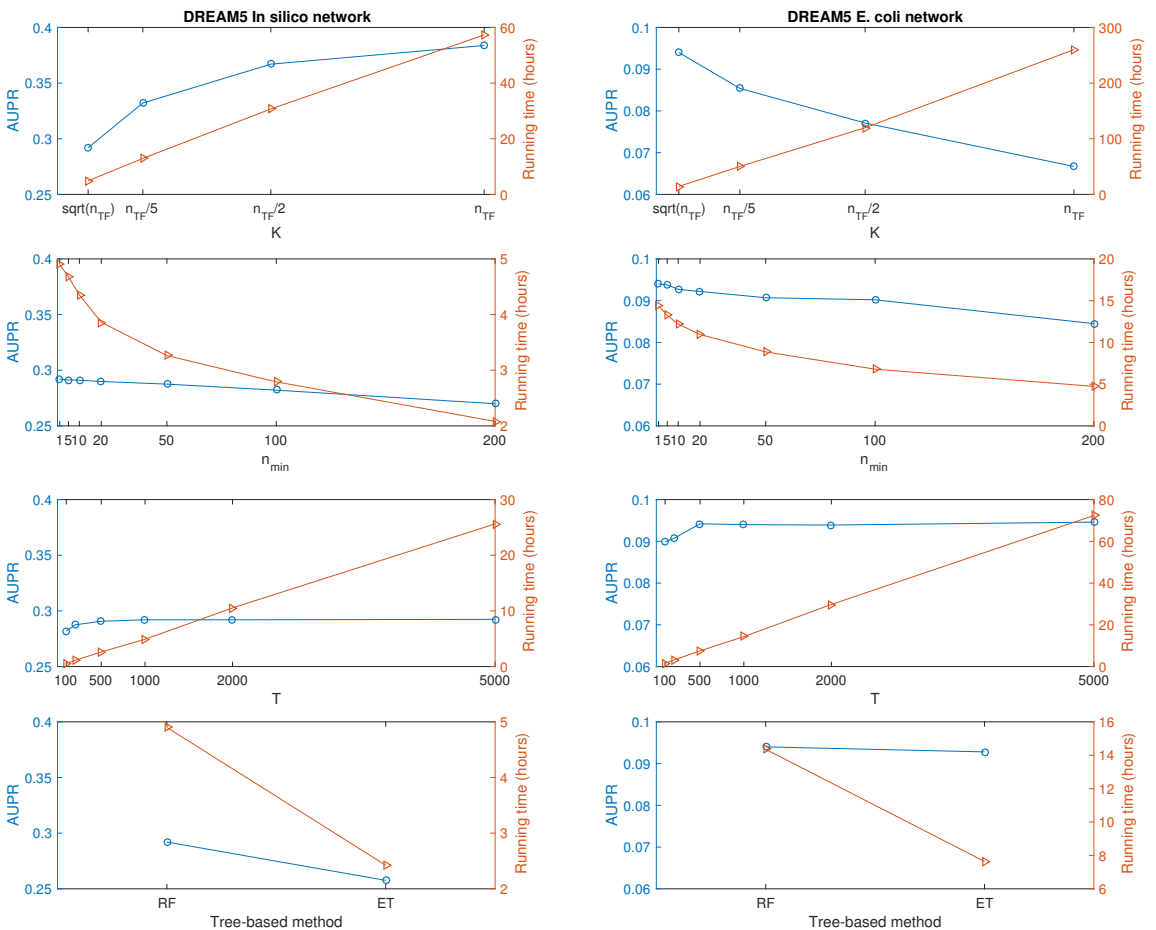

Fig. 4 AUPRs (blue circles) and running times (orange triangles) of GENIE3, when varying the values of the parameters $K$ (number of randomly chosen candidate regulators at each split node of a tree), $n_{\min }$ (minimum number of samples at a leaf) and $T$ (number of trees per ensemble), and when using either Random forest (RF) or Extra-Trees (ET) as tree-based algorithm. When varying the values of one parameter, the values of the remaining parameters were set to their default values. The default values are: $K=\sqrt{n_{T F}}$, where $n_{T F}$ is the number of transcription factors, $n_{\min }=1$, $T=1000$ and the tree-based method is the Random forest algorithm. The results shown in this figure were obtained by using the R implementation of GENIE3. In silico dataset: 805 samples, 1643 genes, 195 transcription factors. E. coli dataset: 805 samples, 4511 genes, 334 transcription factors.

As expected, the predictions are worse when noise is added, for all the values of $K$. In the presence of a high amount of noise, increasing $K$ from $\frac{n_{T F}}{2}$ to $n_{T F}$ results in a (slightly) lower AUPR, a result closer to what is observed for the $E$. coli network. This could be explained by the fact that decreasing the value of $K$ results in predictive tree-based models that overfit less the data and that are therefore more robust to the noise.

The other parameters of GENIE3 have only a minor impact on the performances (Figure 4). In terms of AUPR, the best value of $n_{\min }$, i.e. the minimum number of samples at a leaf, is 1 . Increasing $n_{\min }$ allows to save some computational time, at only a small cost in terms of performances. Regarding the number $T$ of trees per ensemble, we observe that 500 trees already allows to obtain good performances. Further increasing $T$ only results in more computational time, without improving 
Table 2 AUPRs of GENIE3 for the DREAM5 In silico network, when noise is added to the data

\begin{tabular}{lll}
\hline & $\begin{array}{l}\text { Noise } \\
\mathscr{N}(0,0.25)\end{array}$ & $\begin{array}{l}\text { Noise } \\
\mathscr{N}(0,0.5)\end{array}$ \\
\hline$K=\sqrt{n_{T F}}$ & 0.1741 & 0.0435 \\
$K=n_{T F} / 5$ & 0.1893 & 0.0436 \\
$K=n_{T F} / 2$ & 0.2004 & 0.0435 \\
$K=n_{T F}$ & 0.2059 & 0.0426 \\
Random & 0.0125 & 0.0125 \\
\hline
\end{tabular}

the AUPR. Finally, the Extra-Trees algorithm has slightly less good performances than Random forest, but is more computationally efficient.

\subsection{Extensions of GENIE3}

Several methods for GRN inference building on GENIE3 have been developed. The purpose of this section is to give a brief overview of these methods. For more details, the reader can refer to the original articles (referenced in the following subsections).

\subsubsection{Analysis of time series data}

dynGENIE3 (for "dynamical GENIE3") is a variant of GENIE3 that was developed for the analysis of time series of expression data [32]. dynGENIE3 assumes that the expression level $x_{j}$ of gene $g_{j}$ is modelled through the following ordinary differential equation (ODE):

$$
\frac{\mathrm{d} x_{j}(t)}{\mathrm{d} t}=-\alpha_{j} x_{j}(t)+f_{j}(\mathbf{x}(t))
$$

where $\mathbf{x}(t)$ is the vector containing the expressions of all the $G$ genes at time $t$ and $\alpha_{j}$ is a parameter specifying the decay rate of $x_{j}$. In this ODE, it is assumed that the transcription rate of $x_{j}$ is a (potentially non-linear) function $f_{j}$ of the expression levels of the $G$ genes. Like in GENIE3, this function $f_{j}$ is learned in the form of an ensemble of regression trees and the regulators of $g_{j}$ are then identified by computing the variable importance scores derived from the tree model. dynGENIE3 is therefore a semi-parametric approach, as the temporal evolution of each gene expression is modelled with a formal ODE while the transcription function in each ODE is learned in the form of a non-parametric (tree-based) model.

Given the observation time points $t_{1}, t_{2}, \ldots, t_{T}$, the ODE (12) has the following finite approximation:

$$
\begin{aligned}
& \frac{x_{j}\left(t_{k+1}\right)-x_{j}\left(t_{k}\right)}{t_{k+1}-t_{k}}+\alpha_{j} x_{j}\left(t_{k}\right)=f_{j}\left(\mathbf{x}\left(t_{k}\right)\right), \\
& k=1, \ldots, T-1,
\end{aligned}
$$


and the function $f_{j}$ can thus be learned using the following learning sample:

$$
L S^{j}=\left\{\left(\mathbf{x}\left(t_{k}\right), \frac{x_{j}\left(t_{k+1}\right)-x_{j}\left(t_{k}\right)}{t_{k+1}-t_{k}}+\alpha_{j} x_{j}\left(t_{k}\right)\right), k=1, \ldots, T-1\right\} .
$$

The gene decay rates $\alpha_{j}$ in $L S^{j}$ are parameters that are fixed by the user. Their values may be retrieved from the literature, since there exist many studies that experimentally measure the mRNA decay rates in different organisms. However, when such information is not available, a data-driven approach can be used to estimate the $\alpha_{j}$ value directly from the observed expressions $\mathbf{x}_{j}$ of $g_{j}$. For example, a rough estimate of $\alpha_{j}$ can be obtained by assuming an exponential decay $e^{-\alpha_{j} t}$ between the highest and lowest values of $\mathbf{x}_{j}$.

\subsubsection{Analysis of genotype data}

Two extensions of GENIE3 were proposed for the joint analysis of expression and genotype data [33]. It is assumed that we have at our disposal a dataset containing the expression levels of $G$ genes measured in $N$ individuals, as well as the genotype value of one genetic marker for each of these genes in the same $N$ individuals:

$$
D=\left\{\left(\mathbf{x}_{1}, \mathbf{m}_{1}\right),\left(\mathbf{x}_{2}, \mathbf{m}_{2}\right), \ldots,\left(\mathbf{x}_{N}, \mathbf{m}_{N}\right)\right\}
$$

where $\mathbf{x}_{k} \in \mathbb{R}^{G}$ and $\mathbf{m}_{k} \in\{0,1\}^{G}, k=1, \ldots, N$ are respectively the vectors of expression levels and genotype values of the $G$ genes in the $k$-th individual:

$$
\left\{\begin{array}{l}
\mathbf{x}_{k}=\left(x_{k}^{1}, x_{k}^{2}, \ldots, x_{k}^{G}\right)^{\top} \\
\mathbf{m}_{k}=\left(m_{k}^{1}, m_{k}^{2}, \ldots, m_{k}^{G}\right)^{\top}
\end{array}\right.
$$

Note that it is supposed that each genetic marker can have two possible genotype values only ( 0 or 1$)$, as it would be the case for homozygous individuals.

To exploit such data, the first procedure, called GENIE3-SG-joint, assumes that a unique model $f_{j}$ explains the expression of a gene $g_{j}$ in a given individual, knowing the expression levels and the genotype values of the different genes:

$$
x_{k}^{j}=f_{j}\left(\mathbf{x}_{k}^{-j}, \mathbf{m}_{k}\right)+\varepsilon_{k}, \forall k,
$$

where $\varepsilon_{k}$ is a random noise. In the second procedure, called GENIE3-SG-sep, it is assumed that two different models $f_{j}^{x}$ and $f_{j}^{m}$ can both explain the expression of $g_{j}$, either from the expression levels of the other genes, or from the genotype values:

$$
\left\{\begin{array}{l}
x_{k}^{j}=f_{j}^{x}\left(\mathbf{x}_{k}^{-j}\right)+\varepsilon_{k}, \forall k \\
x_{k}^{j}=f_{j}^{m}\left(\mathbf{m}_{k}\right)+\varepsilon_{k}^{\prime}, \forall k
\end{array}\right.
$$

The functions $f_{j}^{e}$ and $f_{j}^{m}$ are therefore respectively learned from two different learning samples. Both GENIE3-SG-joint and GENIE3-SG-sep learn the different func- 
tions $f_{j}$ as ensembles of trees and compute for each candidate regulator $g_{i}$ two scores $w_{i, j}^{x}$ and $w_{i, j}^{m}$, measuring respectively the importances of the expression and of the marker of $g_{i}$ when predicting the expression of $g_{j}$. These two scores are then aggregated, either by a sum or a product, to obtain a single weight $w_{i, j}$ for the regulatory link directed from $g_{i}$ to $g_{j}$.

\subsubsection{Analysis of single-cell data}

GENIE3 is used as such in two frameworks that were developed for the inference of GRNs from single-cell transcriptomic data.

The framework developed by Ocone et al. uses GENIE3 to obtain a prior GRN, which is then refined using an ODE-based approach [34]. The whole procedure allows to identify the GRN as well as the parameters of the ODEs that are used to model the gene expression dynamics.

In the SCENIC framework [35], GENIE3 is used in a first step to identify coexpression modules, i.e. groups of genes that are regulated by the same transcription factor. In a second step, a motif enrichment analysis is performed for each module, and only the modules such that the target genes show an enrichment of a motif of the corresponding transcription factor are retained. The activity of each module in each cell is then evaluated using the single-cell expression data and the activity levels of the different modules are used to perform cell clustering.

\subsubsection{Exploitation of prior knowledge}

The iRafNet method [36] allows to take into account prior information that we have about the network, in the form of prior weights associated with the different network edges. In the original article introducing iRafNet, the prior weights are obtained from diverse types of data, such as protein-protein interaction data or knockout data. To exploit the prior weights, iRafNet uses the same framework as GENIE3, but with a modified version of the Random forest algorithm. At each tree node, instead of randomly sampling $K$ input variables according to a uniform distribution, the $K$ variables are sampled with a bias that favours the variables with a higher prior weight.

\subsubsection{Inference of context-specific networks}

Let us assume that we have different expression datasets respectively related to different contexts (e.g. different pathological conditions). One could then be interested in identifying a GRN that is specific to each of these contexts. To achieve such goal, one could apply a network inference algorithm like GENIE3 to each dataset. However, when the contexts are related (e.g. different subclasses of a cancer), one can expect the different networks to have a lot of commonalities. In this case, approaches 
that jointly analyse the different datasets will potentially yield better performances, as they will assign higher weights to regulatory links that are active in a higher number of contexts. Many of such joint approaches are based on Gaussian graphical models (see e.g. [37-39]). An approach based on trees, called JRF (for "Joint Random Forest") [40], has also been proposed. Given $D$ datasets, JRF consists, for each target gene, in simultaneously learning $D$ tree models. When learning $D$ regression trees in parallel, the idea is to select the same input variable at the same node position in the $D$ different trees. More specifically, for a given input variable $g_{i}$, let $c_{i}^{d}$ be the threshold value that yields the best split at test node $\mathscr{N}$ in the $d$-th tree $(d=1, \ldots, D) . c_{i}^{d}$ is thus the threshold value that maximises the variance reduction $I_{i}^{d}(\mathscr{N})$, as defined in Equation (5), among all the possible threshold values for $g_{i}$. JRF then selects, at node $\mathscr{N}$ in all the $D$ trees, the input variable $g_{i^{*}}$ that maximises:

$$
g_{i^{*}}=\arg \max _{i} \sum_{d=1}^{D} \frac{I_{i}^{d}(\mathscr{N})}{N_{d}},
$$

where $N_{d}$ is the number of samples in the $d$-th dataset. The importance score of a candidate regulator $g_{i}$ in the $d$-th context is then the sum of output variance reductions (as defined in Equation (6)), computed by propagating the samples of the $d$-th dataset in the $d$-th tree model. By selecting, for a given target gene, the same candidate regulators in the $D$ tree models, JRF enforces the similarity between the inferred networks. However, since the importance score of $g_{i}$ in the $d$-th context is computed by using the samples that are related to this context, JRF also allows to identify regulatory links that are active in only one or a few contexts. Note that when there is only one context, JRF is equivalent to GENIE3.

\subsection{Other tree-based approaches}

Besides GENIE3, other tree-based approaches have been proposed for the unsupervised inference of GRNs, which use different types of trees in different frameworks. In [41], networks are reconstructed by learning a single classification tree for each target gene, predicting the state of the gene (up- or down-regulated) from the expression levels of the other genes. In [42], Segal et al. propose a method that partitions the genes into modules, such that genes in the same module have the same regulators and the same regulatory model. The regulatory model of each module is represented by one probabilistic regression tree (where each leaf is associated with a probabilistic distribution of the output variable). Inspired by the work of Segal al., the LeMoNe algorithm [43] also learns module networks, where the regulatory model for each module is in the form of an ensemble of fuzzy decision trees. In [44] M5' model trees are used, i.e. regression trees with linear models at the leaves. In [45], networks are reconstructed by fitting a dynamical model of the gene expressions, where one of the terms of the model is learned in the form of an ensemble of decision trees. Tree-based methods were also used to model expression data jointly 
with other types of data, such as motif counts in the promoter regions of the gene or transcription factor-binding data [46-49]. Another example is [50], which extends the module network procedure to exploit both expression and genotype data.

\section{Discussion}

In this chapter, we presented GENIE3 and other tree-based approaches, that were developed for the inference of gene regulatory networks from expression data. The main advantages of GENIE3 are its non-parametric nature, its ability to detect multivariate interacting effects between candidate regulators and the fact that it has very few parameters. However, like any method, GENIE3 also has its own limitations and could thus be improved along several directions, discussed below.

A first limitation of GENIE3 is that it provides a ranking of the putative regulatory links, rather than a network topology. Since tree-based importance scores are not interpretable from a statistical point of view, choosing a threshold value to distinguish present and absent edges is not a trivial task. Several methods were proposed for addressing the problem of selecting, from tree-based importance scores, the input variables that are relevant for output prediction [51, 52]. These methods could in principle be applied in the context of GENIE3 in order to select the regulators of each target gene. However, most of them are based on multiple - say 1000 - reruns of the tree-based algorithm. If one wishes to incorporate such feature selection approaches into GENIE3, one would need to learn $1000 \times G$ ensembles of trees, where $G$ is the number of genes, which would be impractical for a large value of $G$. Another property of these methods is that they are designed to identify the maximal subset of relevant variables, i.e. all the variables that convey at least some information about the output. For that reason, these methods are not appropriate for the network inference problem. Even if there is no direct edge from gene $g_{i}$ to gene $g_{j}$ in the true network, the expression of $g_{i}$ can still be predictive of the expression of $g_{j}$, through one or several other genes (e.g. $g_{i}$ regulates some gene $g_{k}$, which in turn regulates $g_{j}$ ). In conclusion, each gene of the network is indirectly regulated by (almost) all the other genes, but most of these indirect edges are considered false positives since they are not part of the true network. To avoid the inclusion of such indirect effects, one would need a feature selection method able to determine a minimal subset of variables that convey all the information about an output (and thus make all the other variables conditionally irrelevant). An optimal treatment of this problem would probably require to adopt a more global approach that exploits jointly the $G$ individual rankings related to the different target genes respectively.

GENIE3 could also be improved on the way the variable importance scores are normalised. Given the current normalisation (Equation (11)), the scores of all the putative edges directed towards a given target gene sum up to one. As a consequence, the importance scores that are derived from different tree models are not entirely comparable. For example, let us assume that gene $g_{1}$ has a single regulator $g_{2}$, and that gene $g_{3}$ has two regulators $g_{4}$ and $g_{5}$. With a sufficient amount of data, GENIE3 
will assign a score of 1 to the edge $g_{2} \rightarrow g_{1}$, but a score of only 0.5 to $g_{4} \rightarrow g_{3}$ and $g_{5} \rightarrow g_{3}$. An optimal way of normalising the importance score is thus still needed at this stage.

So far, GENIE3 has only been evaluated in an empirical way. It would however be interesting to better characterise the method - in particular the tree-based importance scores used within - from a theoretical point of view. This would actually constitute an important contribution in the machine learning field, as there has been very few works focusing on the theoretical analysis of the importance measures derived from tree ensembles [53-55].

Despite the good scalability of GENIE3 with respect to some families of methods (such as methods based on differential equations or Bayesian methods), a substantial amount of time is still needed to reconstruct a large network (e.g. it takes 20 hours to reconstruct the $E$. coli network from 805 samples, see Table 1). With the recent developments in single-cell RNA-seq technologies, datasets with a size of the order of $100 \mathrm{~K}$ cells are becoming available. Speeding up the GENIE3 algorithm would be necessary if one wishes to apply it on such large datasets.

Acknowledgements VAHT is a Post-doctoral Fellow of the F.R.S.-FNRS.

\section{References}

[1] Geurts P, Irrthum A, Wehenkel L (2009) Supervised learning with decision tree-based methods in computational and systems biology. Mol Biosyst 5(12):1593-605

[2] Boulesteix AL, Janitza S, Kruppa J, König IR (2012) Overview of random forest methodology and practical guidance with emphasis on computational biology and bioinformatics. Wiley Interdisciplinary Reviews: Data Mining and Knowledge Discovery 2(6):493-507

[3] Biau G, Scornet E (2016) A random forest guided tour. TEST 25(2):197-227

[4] Huynh-Thu VA, Irrthum A, Wehenkel L, Geurts P (2010) Inferring regulatory networks from expression data using tree-based methods. PLoS ONE 5(9):e12,776

[5] Marbach D, Costello JC, Küffner R, Vega N, Prill RJ, Camacho DM, Allison KR, the DREAM5 Consortium, Kellis M, Collins JJ, Stolovitzky G (2012) Wisdom of crowds for robust gene network inference. Nature Methods 9(8):796-804

[6] Omranian N, Eloundou-Mbebi JMO, Mueller-Roeber B, Nikoloski Z (2016) Gene regulatory network inference using fused lasso on multiple data sets. Scientific Reports 6:20,533

[7] Kiani NA, Zenil H, Olczak J, Tegnér J (2016) Evaluating network inference methods in terms of their ability to preserve the topology and complexity of genetic networks. Seminars in Cell \& Developmental Biology 51:44 - 52 
[8] Bellot P, Olsen C, Salembier P, Oliveras-Vergés A, Meyer PE (2015) NetBenchmark: a bioconductor package for reproducible benchmarks of gene regulatory network inference. BMC Bioinformatics 16:312

[9] Maetschke SR, Madhamshettiwar PB, Davis MJ, Ragan MA (2014) Supervised, semi-supervised and unsupervised inference of gene regulatory networks. Briefings in Bioinformatics 15(2):195-211

[10] Zhang X, Liu K, Liu ZP, Duval B, Richer JM, Zhao XM, Hao JK, Chen L (2013) NARROMI: a noise and redundancy reduction technique improves accuracy of gene regulatory network inference. Bioinformatics 29(1):106-113

[11] Feizi S, Marbach D, Médard M, Kellis M (2013) Network deconvolution as a general method to distinguish direct dependencies in networks. Nature Biotechnology 31:726-733

[12] Madhamshettiwar PB, Maetschke SR, Davis MJ, Reverter A, Ragan MA (2012) Gene regulatory network inference: evaluation and application to ovarian cancer allows the prioritization of drug targets. Genome Medicine 4(5):41

[13] Qi J, Michoel T (2012) Context-specific transcriptional regulatory network inference from global gene expression maps using double two-way t-tests. Bioinformatics 28(18):2325-2332

[14] Imam S, Noguera DR, Donohue TJ (2015) An integrated approach to reconstructing genome-scale transcriptional regulatory networks. PLoS Computational Biology 11(2):e1004,103

[15] Arrieta-Ortiz ML, Hafemeister C, Bate AR, Chu T, Greenfield A, Shuster B, Barry SN, Gallitto M, Liu B, Kacmarczyk T, Santoriello F, Chen J, Rodrigues CD, Sato T, Rudner DZ, Driks A, Bonneau R, Eichenberger P (2015) An experimentally supported model of the bacillus subtilis global transcriptional regulatory network. Molecular Systems Biology 11(11)

[16] Carrera J, Estrela R, Luo J, Rai N, Tsoukalas A, Tagkopoulos I (2014) An integrative, multi-scale, genome-wide model reveals the phenotypic landscape of escherichia coli. Molecular Systems Biology 10(7)

[17] Sabaghian E, Drebert Z, Inzé D, Saeys Y (2015) An integrated network of Arabidopsis growth regulators and its use for gene prioritization. Scientific Reports 5:17,617

[18] Taylor-Teeples M, Lin L, de Lucas M, Turco G, Toal TW, Gaudinier A, Young NF, Trabucco GM, Veling MT, Lamothe R, Handakumbura PP, Xiong G, Wang C, Corwin J, Tsoukalas A, Zhang L, Ware D, Pauly M, Kliebenstein DJ, Dehesh K, Tagkopoulos I, Breton G, Pruneda-Paz JL, Ahnert SE, Kay SA, Hazen SP, Brady SM (2015) An Arabidopsis gene regulatory network for secondary cell wall synthesis. Nature 517(7536):571-575

[19] Marchand G, Huynh-Thu VA, Kane N, Arribat S, Varès D, Rengel D, Balzergue S, Rieseberg L, Vincourt P, Geurts P, Vignes M, Langlade NB (2014) Bridging physiological and evolutionary time-scales in a gene regulatory network. New Phytologist 203(2):685-696

[20] Potier D, Davie K, Hulselmans G, NavalSanchez M, Haagen L, Huynh-Thu V, Koldere D, Celik A, Geurts P, Christiaens V, Aerts S (2014) Mapping gene reg- 
ulatory networks in Drosophila eye development by large-scale transcriptome perturbations and motif inference. Cell Reports 9(6):2290-2303

[21] Jo J, Hwang S, Kim HJ, Hong S, Lee JE, Lee SG, Baek A, Han H, Lee JI, Lee I, Lee DR (2016) An integrated systems biology approach identifies positive cofactor 4 as a factor that increases reprogramming efficiency. Nucleic Acids Research 44(3):1203-1215

[22] Acquaah-Mensah GK, Taylor RC (2016) Brain in situ hybridization maps as a source for reverse-engineering transcriptional regulatory networks: Alzheimer's disease insights. Gene 586(1):77 - 86

[23] Verfaillie A, Imrichova H, Atak ZK, Dewaele M, Rambow F, Hulselmans G, Christiaens V, Svetlichnyy D, Luciani F, Van den Mooter L, Claerhout S, Fiers M, Journe F, Ghanem GE, Herrmann C, Halder G, Marine JC, Aerts S (2015) Decoding the regulatory landscape of melanoma reveals teads as regulators of the invasive cell state. Nature Communications 6:6683

[24] Ko JH, Gu W, Lim I, Zhou T, Bang H (2014) Expression profiling of mitochondrial voltage-dependent anion channel-1 associated genes predicts recurrencefree survival in human carcinomas. PLoS ONE 9(10):e110,094

[25] Hastie T, Tibshirani R, Friedman J (2009) The elements of statistical learning: data mining, inference and prediction, 2nd edn. Springer

[26] Bishop CM (2006) Pattern Recognition and Machine Learning. Springer

[27] Breiman L, Friedman JH, Olsen RA, Stone CJ (1984) Classification and Regression Trees. Wadsworth International (California)

[28] Breiman L (1996) Bagging predictors. Machine Learning 24(2):123-140

[29] Breiman L (2001) Random forests. Machine Learning 45(1):5-32

[30] Geurts P, Ernst D, Wehenkel L (2006) Extremely randomized trees. Machine Learning 36(1):3-42

[31] Strobl C, Boulesteix AL, Zeileis A, Horthorn T (2007) Bias in random forest variable importance measures: Illustrations, sources and a solution. BMC Bioinformatics 8:25

[32] Huynh-Thu VA, Geurts P (2018) dynGENIE3: dynamical GENIE3 for the inference of gene networks from time series expression data. Scientific Reports $8(1): 3384$

[33] Huynh-Thu VA, Wehenkel L, Geurts P (2013) Gene regulatory network inference from systems genetics data using tree-based methods. In: de la Fuente A (ed) Gene Network Inference - Verification of Methods for Systems Genetics Data, Springer, pp 63-85

[34] Ocone A, Haghverdi L, Mueller NS, Theis FJ (2015) Reconstructing gene regulatory dynamics from high-dimensional single-cell snapshot data. Bioinformatics 31(12):i89-i96

[35] Aibar S, González-Blas CB, Moerman T, Huynh-Thu VA, Imrichova H, Hulselmans G, Rambow F, Marine JC, Geurts P, Aerts J, van den Oord J, Atak ZK, Wouters J, Aerts S (2017) SCENIC: single-cell regulatory network inference and clustering. Nature Methods 14:1083-1086

[36] Petralia F, Wang P, Yang J, Tu Z (2015) Integrative random forest for gene regulatory network inference. Bioinformatics 31(12):i197-i205 
[37] Chiquet J, Grandvalet Y, Ambroise C (2011) Inferring multiple graphical structures. Statistics and Computing 21(4):537-553

[38] Mohan K, London P, Fazel M, Witten D, Lee SI (2014) Node-based learning of multiple gaussian graphical models. J Mach Learn Res 15(1):445-488

[39] Tian D, Gu Q, Ma J (2016) Identifying gene regulatory network rewiring using latent differential graphical models. Nucleic Acids Research 44(17):e140

[40] Petralia F, Song WM, Tu Z, Wang P (2016) New Method for Joint Network Analysis Reveals Common and Different Coexpression Patterns among Genes and Proteins in Breast Cancer. Journal of Proteome Research 15(3):743-754

[41] Soinov LA, Krestyaninova MA, Brazma A (2003) Towards reconstruction of gene networks from expression data by supervised learning. Genome Biology 4(1):R6

[42] Segal E, Shapira M, Regev A, Pe'er D, Botstein D, Koller D, Friedman N (2003) Module networks: identifying regulatory modules and their conditionspecific regulators from gene expression data. Nature Genetics 34:166-176

[43] Joshi A, De Smet R, Marchal K, Van de Peer Y, Michoel T (2009) Module networks revisited: computational assessment and prioritization of model predictions. Bioinformatics 25(4):490-496

[44] Nepomuceno-Chamorro IA, Aguilar-Ruiz JS, Riquelme JC (2010) Inferring gene regression networks with model trees. BMC Bioinformatics 11:517

[45] Huynh-Thu VA, Sanguinetti G (2015) Combining tree-based and dynamical systems for the inference of gene regulatory networks. Bioinformatics 31(10):1614-1622

[46] Middendorf M, Kundaje A, Wiggins C, Freund Y, Leslie C (2004) Predicting genetic regulatory response using classification. Bioinformatics 20(suppl_1):i232-i240

[47] Phuong TM, Lee D, Lee KH (2004) Regression trees for regulatory element identification. Bioinformatics 20(5):750-757

[48] Ruan J, Zhang W (2006) A bi-dimensional regression tree approach to the modeling of gene expression regulation. Bioinformatics 22(3):332-340

[49] Xiao Y, Segal MR (2009) Identification of yeast transcriptional regulation networks using multivariate random forests. PLOS Computational Biology 5(6): 1000,414

[50] Lee SI, Pe'er D, Dudley AM, Church GM, Koller D (2006) Identifying regulatory mechanisms using individual variation reveals key role for chromatin modification. Proceedings of the National Academy of Sciences 103(38):14,062-14,067

[51] Huynh-Thu VA, Saeys Y, Wehenkel L, Geurts P (2012) Statistical interpretation of machine learning-based feature importance scores for biomarker discovery. Bioinformatics 28(13):1766-1774

[52] Degenhardt F, Seifert S, Szymczak S (2017) Evaluation of variable selection methods for random forests and omics data sets. Briefings in Bioinformatics $p$ bbx 124

[53] Ishwaran H (2007) Variable importance in binary regression trees and forests. Electron J Statist 1:519-537 
[54] Louppe G, Wehenkel L, Sutera A, Geurts P (2013) Understanding variable importances in forests of randomized trees. In: Burges CJC, Bottou L, Welling M, Ghahramani Z, Weinberger KQ (eds) Advances in Neural Information Processing Systems 26, Curran Associates, Inc., pp 431-439

[55] Sutera A, Louppe G, Huynh-Thu VA, Wehenkel L, Geurts P (2016) Contextdependent feature analysis with random forests. In: Proceedings of the ThirtySecond Conference on Uncertainty in Artificial Intelligence, AUAI Press, UAI'16, pp 716-725 